PAPER

\section{Structured targets for advanced laser-driven} sources

To cite this article: L Fedeli et al 2018 Plasma Phys. Control. Fusion 60014013

View the article online for updates and enhancements.

\section{Related content}

- $\frac{\text { High field plasmonics and laser-plasma }}{\text { acceleration in solid targets }}$
A Sgattoni, L Fedeli, G Cantono et al.
- Development of foam-based layered
$\frac{\text { targets for laser-driven ion beam }}{\text { production }}$
I Prencipe, A Sgattoni, D Dellasega et al.
- Intense terahertz radiation from relativistic
$\frac{\text { laser-plasma interactions }}{\text { G Q Liao, Y T Li, C Li et al. }}$




\title{
Structured targets for advanced laser-driven sources
}

\author{
L Fedeli ${ }^{1}$, A Formenti ${ }^{1}$, L Cialfi ${ }^{1}$, A Sgattoni $^{2,3}$, G Cantono $^{4,5,6,7}$ and \\ M Passoni ${ }^{1}$ \\ ${ }^{1}$ Dipartimento di Energia, Politecnico di Milano, Italy \\ ${ }^{2}$ LULI-UPMC: Sorbonne Universities, CNRS, École Polytechnique, CEA, F-75013 Paris, France \\ ${ }^{3}$ LESIA, Observatoire de Paris, CNRS, UPMC: Sorbonne Universities, F-92195 Meudon, France \\ ${ }^{4}$ LIDYL, CEA, CNRS, Universit Paris-Saclay, CEA Saclay, F-91191 Gif-sur-Yvette, France \\ ${ }^{5}$ Universit Paris Sud, Paris, F-91400 Orsay, France \\ ${ }^{6}$ National Institute of Optics, National Research Council (CNR/INO) A.Gozzini unit, I-56124 Pisa, Italy \\ ${ }^{7}$ Enrico Fermi Department of Physics, University of Pisa, I-56127 Pisa, Italy
}

E-mail: luca.fedeli@polimi.it

Received 26 June 2017, revised 8 August 2017

Accepted for publication 4 September 2017

Published 6 November 2017

\begin{abstract}
Structured targets offer great control over ultra-intense laser-plasma interaction, allowing the optimization of laser-target coupling for specific applications. By means of particle-in-cell simulations we investigated three applications in particular: high-order harmonic generation (HHG) with grating targets, enhanced target coupling with multilayer targets and the generation of intense laser-driven terahertz $(\mathrm{THz})$ pulses with structured targets. The irradiation of a solid grating target at the resonance angle for surface plasmon excitation enhances the HHG with respect to flat targets. Multilayer targets consisting of solid foils coated with a very low-density near-critical layer lead to a strong laser absorption and hot electron production that can improve laser-driven ion acceleration. We also explored the generation of $\mathrm{THz}$ radiation showing how using either gratings or multilayer targets the emission can be strongly enhanced with respect to simple flat targets.
\end{abstract}

Keywords: relativistic laser-plasma interaction, high-order harmonic generation, structured targets, laser-driven ion acceleration, laser-driven terahertz generation, particle-in-cell simulations

(Some figures may appear in colour only in the online journal)

\section{Introduction}

The realization of laser-driven ultra-intense radiation sources is one of the main goals of the research activities carried out in the field of relativistic laser-matter interaction. Various schemes have been proposed for a number of applications, including high-energy particle sources (electrons [1] and ions $[2,3])$, high intensity photon sources [4], high-order harmonic generation (HHG) [5] and ultra-intense terahertz (THz) sources [6]. In parallel with the development of these applications, several advanced target concepts [7] have been investigated, aiming at an improvement or a better control over the aforementioned schemes.
In this work we review some recent numerical results obtained with two specific advanced targets: relativistic surfaceplasmon enhanced HHG with gratings [8](section 2), electron heating and enhanced ion acceleration with multilayer targets [9-11](section 3). Moreover, we provide an exploratory numerical investigation of laser-driven $\mathrm{THz}$ generation exploiting the aforementioned target concepts (section 4). THz emission from solid targets irradiated at relativistic or sub-relativistic intensities has been correlated with the conversion efficiency of laser energy into hot electrons energy [12]. Since both grating targets $[13,14]$ and multilayer targets $[11,15]$ generally provide a significantly higher laser absorption with respect to simple flat targets, an enhancement of $\mathrm{THz}$ emission may also be expected. 


\section{Enhanced HHG with grating targets}

Grating targets have been investigated for a wide variety of applications, including laser-driven ion acceleration [13, 14, 16], synchrotron emission [17], electron acceleration [18, 19] and control [20, 21] or enhancement [8, 22-25] of HHG from lasersolid interaction. Here we review a recent numerical work on HHG using solid grating targets, where we observed that the irradiation of a grating at the resonance angle for surface plasmon excitation enhances HHG with respect to a flat solid target [8].

HHG with relativistic laser pulses interacting with solid targets $[5,26-30]$ is due to the relativistic motion of the electrons in the intense laser field at the target surface $[31,32]$ and has been proposed as a way to achieve higher intensities of the emitted harmonics with respect to conventional schemes based on atomic recollision in gaseous targets [33]. Indeed, HHG generation with gas targets is ultimately limited by the ionization threshold $\left(I \sim 10^{15} \mathrm{~W} \mathrm{~cm}^{-2}\right)$, while such intensity limit does not apply for HHG with laser-solid interaction. The use of a grating as a target for HHG has been proposed as a strategy to angularly separate the emitted harmonics, in order to obtain a quasi-monochromatic extreme ultra-violet (XUV) source [24, 25, 34] (the harmonic orders between the 8th and the 80th of a Ti:Sapphire laser with a wavelength $\sim 800 \mathrm{~nm}$ lie in the XUV spectral region). Given a grating with a spacing $d$ between the grooves, the $m$ th harmonic order is diffracted according to [21, 22]:

$$
n \lambda / m d=\sin \left(\theta_{\mathrm{i}}\right)+\sin \left(\theta_{m n}\right),
$$

where $\lambda$ is the laser wavelength, $n$ is the diffraction order, $\theta_{\mathrm{i}}$ is the angle of incidence of the laser pulse and $\theta_{m n}$ is the diffraction angle.

When focusing a laser pulse on a grating target, a surface plasmon can be excited if a matching condition between the grating period and the angle of incidence is met. Experimental evidence of the excitation of surface plasmons in laser-grating interaction at relativistic intensities has been recently provided $[13,19,35]$. Since surface plasmon excitation is associated with electromagnetic field enhancement at the target surface, an enhancement of HHG can be expected, in addition to the grating diffraction effects.

Neglecting relativistic effects, for a cold, dense plasma (i.e. plasma frequency $\omega_{\mathrm{p}} \gg 2 \pi c / \lambda$ ), the resonance angle $\theta_{\text {res }}$ for surface plasmon excitation is given by [36]:

$$
j \lambda / d=1+\sin \left(\theta_{\text {res }}\right),
$$

where $j$ is an integer. Even though equation (2) has been derived in a purely non-relativistic theory, recent works $[13,19,35]$ suggest that this condition should hold even at relativistic laser intensities.

The irradiation of a grating target in a configuration suitable for the excitation of surface plasmons has been considered in [8], by means of 2D numerical simulations performed with the open source particle-in-cell (PIC) code piccante $[37,38]$. A box size of $80 \lambda \times 80 \lambda$ and a resolution up to $\lambda / 400$ were used. The laser had a normalized intensity $\mathrm{a}_{0}=15$ and a temporal duration of $12 \lambda / \mathrm{c}$ (intensity FWHM). The target was a $1 \lambda$ thick grating with a spacing $d=2 \lambda$, which according to equation (2) corresponds to a resonance angle $\theta_{\text {res }}=30^{\circ}$. The peak-to-valley depth of the grooves was $0.25 \lambda$. A simple flat target was also simulated for comparison. The electron density of the targets was $n_{\mathrm{e}}=128 n_{\mathrm{c}}$, sampled with 144 macro-particles per cell $\left(n_{\mathrm{c}}=\frac{\pi m_{\mathrm{e}} c^{2}}{\lambda^{2} e^{2}}\right.$ is the critical electron density ( $e$ is the elementary charge, $m_{\mathrm{e}}$ is the electron rest mass and $c$ is the speed of light). In the simulations the targets were irradiated at various incidence angles $\left(15^{\circ}-45^{\circ}\right)$ and a Fourier transform of the diffracted electromagnetic field was performed in order to extract the harmonic content.

Figure 1(a) shows the $\hat{z}$ component of the magnetic field before and after the interaction with the grating target. Figure 1(b) shows the Fourier transform of the $\hat{z}$ component of the magnetic field in the $x>0$ half-plane for a flat target irradiated at $45^{\circ}$ and for a grating target irradiated at $\theta_{\mathrm{i}}=$ $15^{\circ}, 30^{\circ}, 35^{\circ}$ and $45^{\circ}$. In the case of a flat target all the harmonic orders are emitted along the specular direction, while for the grating high-order harmonics are diffracted, according to equation (1). A strong enhancement of harmonic emission is observed at incidence angles close to $30^{\circ}$, the expected surface plasmon resonance angle, in particular for harmonic orders diffracted close to the target surface $\left(k_{x}=0\right)$.

Finally, figure 1(c) shows a comparison between the spectrum of the emission collected within $45^{\circ} \pm 2.5^{\circ}$ for the flat target irradiated at $45^{\circ}$ and that of the emission collected within $80^{\circ} \pm 2.5^{\circ}$ for the grating target irradiated at $35^{\circ}$ (these cases were observed to provide the highest harmonic yield for the two target types). While the spectrum for the flat target is dominated by the $m=1$ order (i.e. the reflected laser light), for the grating a suppression of the $m=1$ order and a significant increase of the intensity of emitted harmonics is observed. The enhancement of the harmonic emission is particularly evident for higher harmonic orders ( $\approx 2$ orders of magnitude for $m=40$ ).

These results show that the implementation of the described scheme in a laser-plasma interaction experiment should allow to observe higher-order harmonics irradiating a grating target near-resonance than a simple flat target. This approach may find application as an ultra-intense, quasimonochromatic, XUV source.

\section{Foam-attached foils for enhanced electron heating and ion acceleration}

Multilayer targets consisting in solid foils coated with a lowdensity layer [39-41] have been investigated within the framework of laser-driven ion acceleration [2, 3], where they have been proven to optimize the process. Specifically, they can lead to an increase of both the energy and the number of accelerated ions with respect to flat solid foils [9-11, 15, 41-43]. This could be beneficial for several potential applications [44, 45], such as radiotherapy [46, 47], material science $[48,49]$ or ultra-fast neutron sources [50].

Here we review some recent results concerning a numerical investigation of electron heating with multilayer targets [11], which is strongly related to the ion acceleration process $[2,3]$.

The use of a solid foil coupled with a low-density layernear-critical when fully ionized-has been proposed as a 


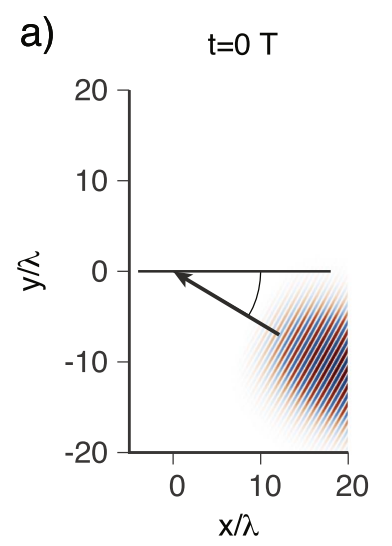

b)

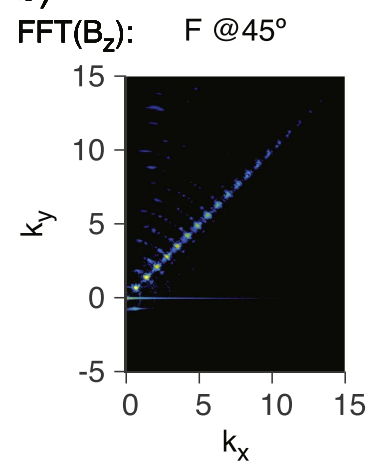

$\mathrm{t}=35 \mathrm{~T}$

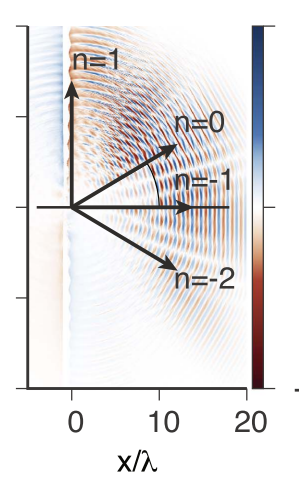

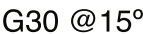

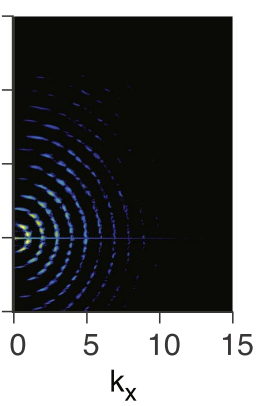

c)

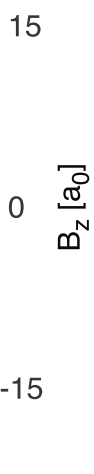

G30@30

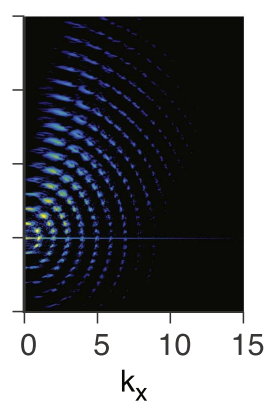

harmonic emission $(\mathrm{t}=35 \mathrm{~T})$

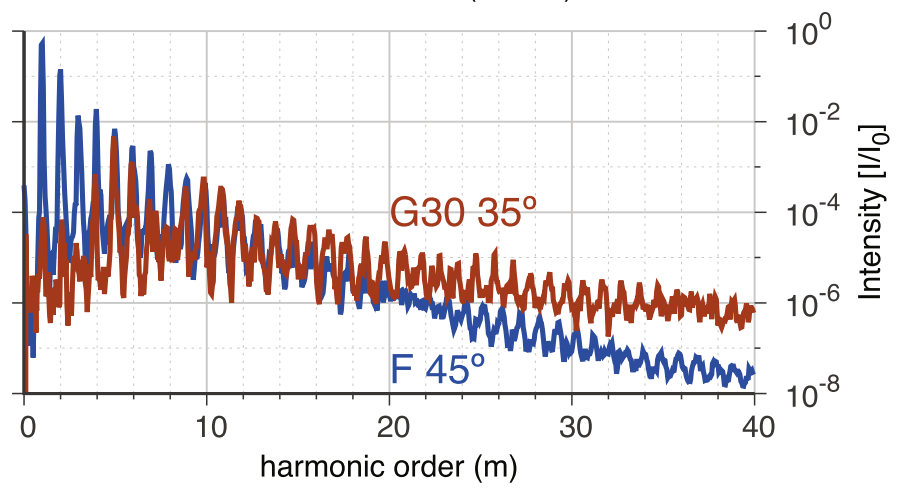

\section{G30@45}

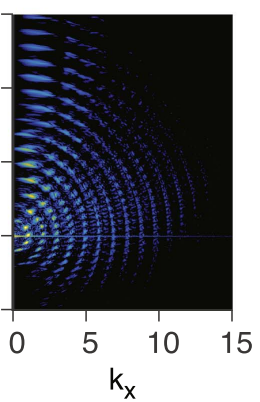

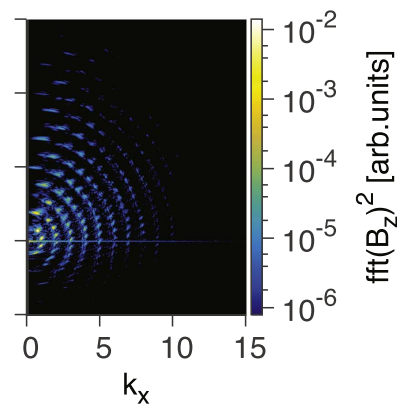

Figure 1. Resonant enhancement of HHG with grating targets. (a) $\hat{z}$ component of the magnetic field before and after the interaction with the grating target (expected resonance at $30^{\circ}$ ). (b) Fourier transform of $B_{z}$ for a flat target irradiated at $45^{\circ}$ and for the grating target irradiated at $15^{\circ}, 30^{\circ}, 35^{\circ}, 45^{\circ}$. (c) Spectrum of the emitted radiation collected by a synthetic detector at $45^{\circ} \pm 2.5^{\circ}$ and $80^{\circ} \pm 2.5^{\circ}$ for the grating target.

possible solution to increase laser-target coupling [15, 51]. Indeed, laser interaction with a plasma at density $n_{\mathrm{e}} \sim n_{\mathrm{c}}$ is characterized by strong absorption and several nonlinear effects such as channel formation [52], self-focusing [52] and high-energy particle acceleration [53].

Experimental and numerical investigations of laser-driven ion acceleration with near-critical materials [9, 10, 41-43] reported a strong enhancement of the energy and the number of accelerated ions under certain target parameters. In $[9,10,43]$ the effect was attributed to an enhanced target normal sheath acceleration (TNSA) mechanism due to an increase of the energy and of the number of electrons accelerated towards the back side of the target, while in [41] authors claimed an enhanced radiation pressure acceleration mechanism due to self-focusing and steepening of the laser pulse in the near-critical plasma.

Near-critical layers in $[9,10,43]$, were obtained coating a thin solid foil with a very low-density nanostructured carbon foam [40]), whereas in [41, 42] authors exploited nanotubes and plastic foams, respectively. Regardless of the technique, while near-critical on average, all these targets are constituted by alternating voids and solid-density nanostructures. Due to the extreme contrast of modern day ultra-short laser systems [54], these nanostructures can survive long enough to influence the interaction with the laser.

In [11] an extensive numerical campaign was carried out in order to elucidate the electron heating process in multilayer targets, taking into account a possible role played by the nanostructure of the near-critical layer. In this work 2D numerical simulations were performed with piccante code $[37,38]$. Simulated targets consisted in $0.5 \mu \mathrm{m}$ thick preionized $(Z / A=1 / 3)$ foils with an electron density $n_{\mathrm{e}}=80 n_{\mathrm{c}}$, coated with a $8 \mu \mathrm{m}$ thick 'foam' layer with an average electron density equal to $1 n_{\mathrm{c}}(Z / A=1 / 2)$. Two different types of foam were compared: a 'homogeneous' and a 'nanostructured' near-critical layer consisting in a spatially random collection of $10 \mathrm{~nm}$ over-dense $\left(100 n_{\mathrm{c}}\right)$ spheres, with a filling factor of $1 \%$ (so that the average density of the plasma was still $\left.1 n_{\mathrm{c}}\right)$. The laser pulse $(\lambda=0.8 \mu \mathrm{m})$ was P-polarized, Gaussian shaped in the transverse direction (waist of $3 \mu \mathrm{m}$ ) with $\sin ^{2}$ temporal envelope (intensity FWHM of $25 \mathrm{fs}$ ). The peak intensity was varied between the normalized laser amplitudes $a_{0}=1.5$ and $a_{0}=15$ (i.e. from a table-top multi-TW facility up to a few hundred s TW facility [55]). The angle of incidence was $30^{\circ}$. As reported in figure 2 , laser interaction with foam-attached targets, either modelled with a homogeneous or nanostructured near-critical layer, results in an enhanced laser-matter coupling, leading to a significant increase of the electron temperature with respect to simple foils. The electron temperature was found to be lower for the nanostructured target than for the homogeneous one. This might be due to the fact that a fraction of the total energy is lost to the ion population in the Coulomb explosion of the nanospheres. A similar effect was observed in [56], where an extensive parametric investigation of laser interaction with 

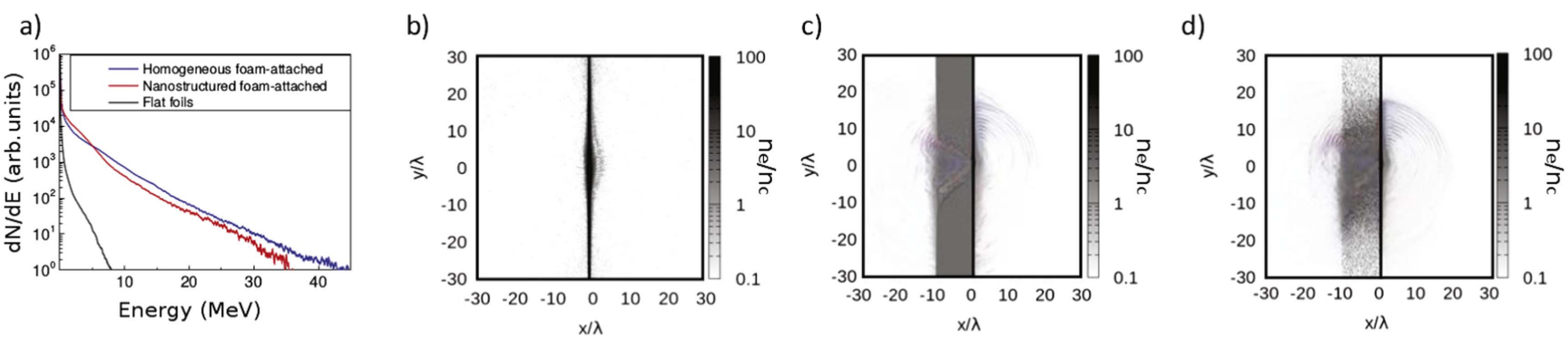

Figure 2. Laser interaction with flat foils and foam-attached targets. (a) Electron energy spectra taken when the kinetic energy of the electron population reaches its maximum for a flat foil (black), a nanostructured foam-attached target (red) and a homogeneous foam-attached target (blue). (b)-(d) Electron density normalized with respect to the critical density for a flat foil, homogeneous foam-attached target and nanostructured foam-attached target, respectively. The snapshots were taken at the same temporal frame of panel (a). The $\hat{z}$ component of the magnetic field is superimposed to the density plot in blue-red scale.
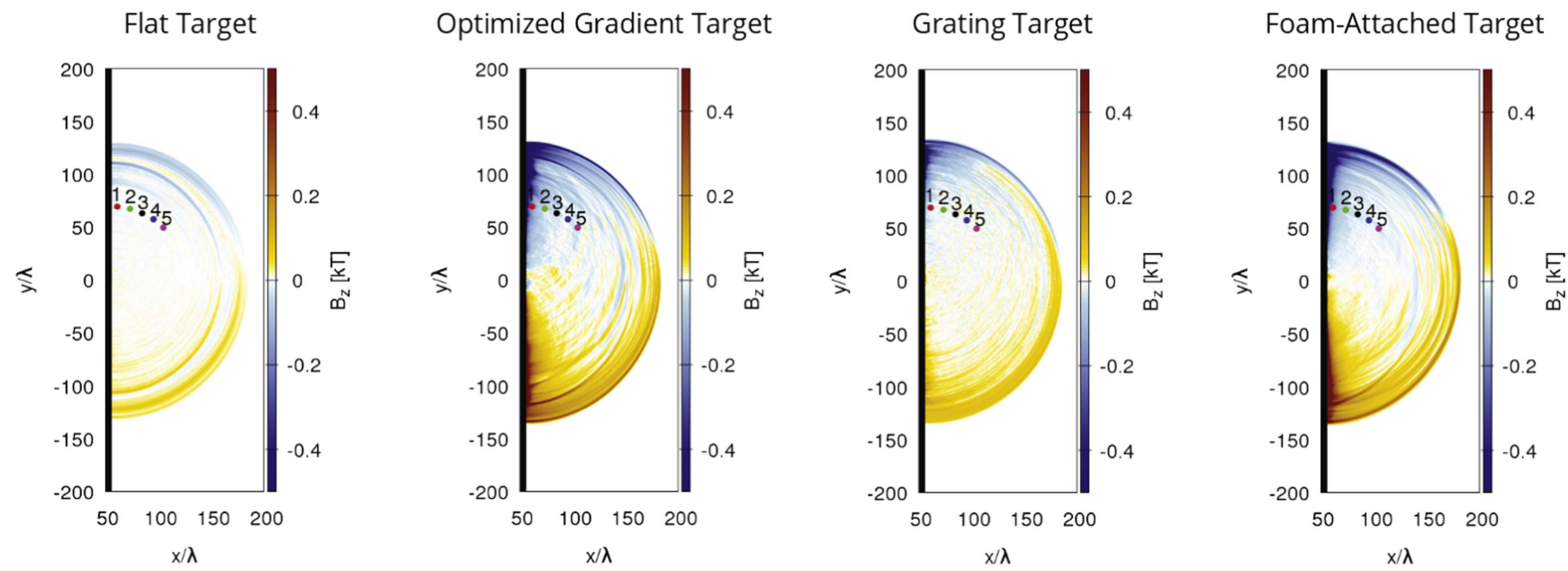

Figure 3. The $B_{z}$ field component at $t=200 \lambda / c$ averaged over one laser cycle for the flat target, the target with an exponential density gradient optimized for resonant absorption, the grating target and the foam-attached target described in section 4 . The numbered dots show the position of 'probe' diagnostics, which collect the value of the electromagnetic field as a function of time in a given position. Probes are positioned at a $70 \lambda$ distance from the target centre, at $85^{\circ}, 75^{\circ}, 65^{\circ}, 55^{\circ}$ and $45^{\circ}$ from target normal, respectively. Field intensities are shown in kiloTesla units (for the conversion from code units, a laser wavelength $\lambda=0.8 \mu \mathrm{m}$ was considered).

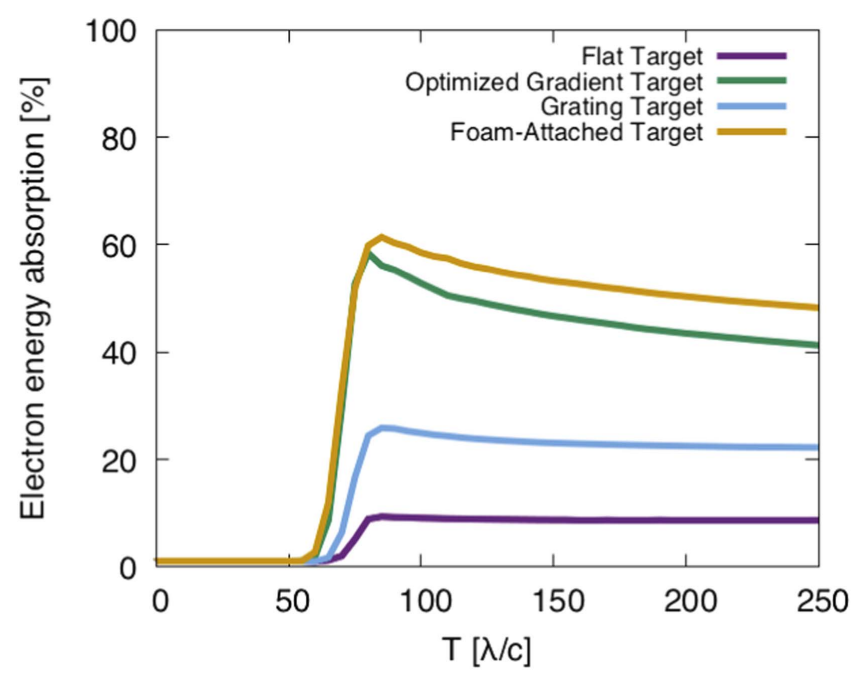

Figure 4. Absorption of laser energy into electron kinetic energy as a function of time for all the cases simulated in section 4 . After reaching a peak at $t \approx 75 \lambda / c$, the energy of the electron population decreases over time due to energy absorbed by the ion acceleration process. nanostructured near-critical plasmas was carried out. In order to benchmark the results of this numerical campaign with experimental data, the electron temperatures obtained from the simulations were coupled with simple models of TNSA [57], providing an estimation for the maximum ion energies. Estimations obtained using the electron temperature of the nanostructured case proved to be in close agreement with recent experimental results $[9,10]$, while the higher temperatures obtained with the uniform foam targets lead to an overestimation of the ions maximum energies. The higher electron temperatures obtained with foam-attached targets with respect to simple flat foils justify the higher ion energies observed in the experiments with the formers.

We remark that simulations with foam-attached targets in [11] were performed with a relatively small angle of incidence of $30^{\circ}$. However, results in [15] provide an indirect indication that foam-attached targets could still allow for a significant enhancement of electron heating with respect to flat solid foils even for larger incidence angles (up to $60^{\circ}$ ). 

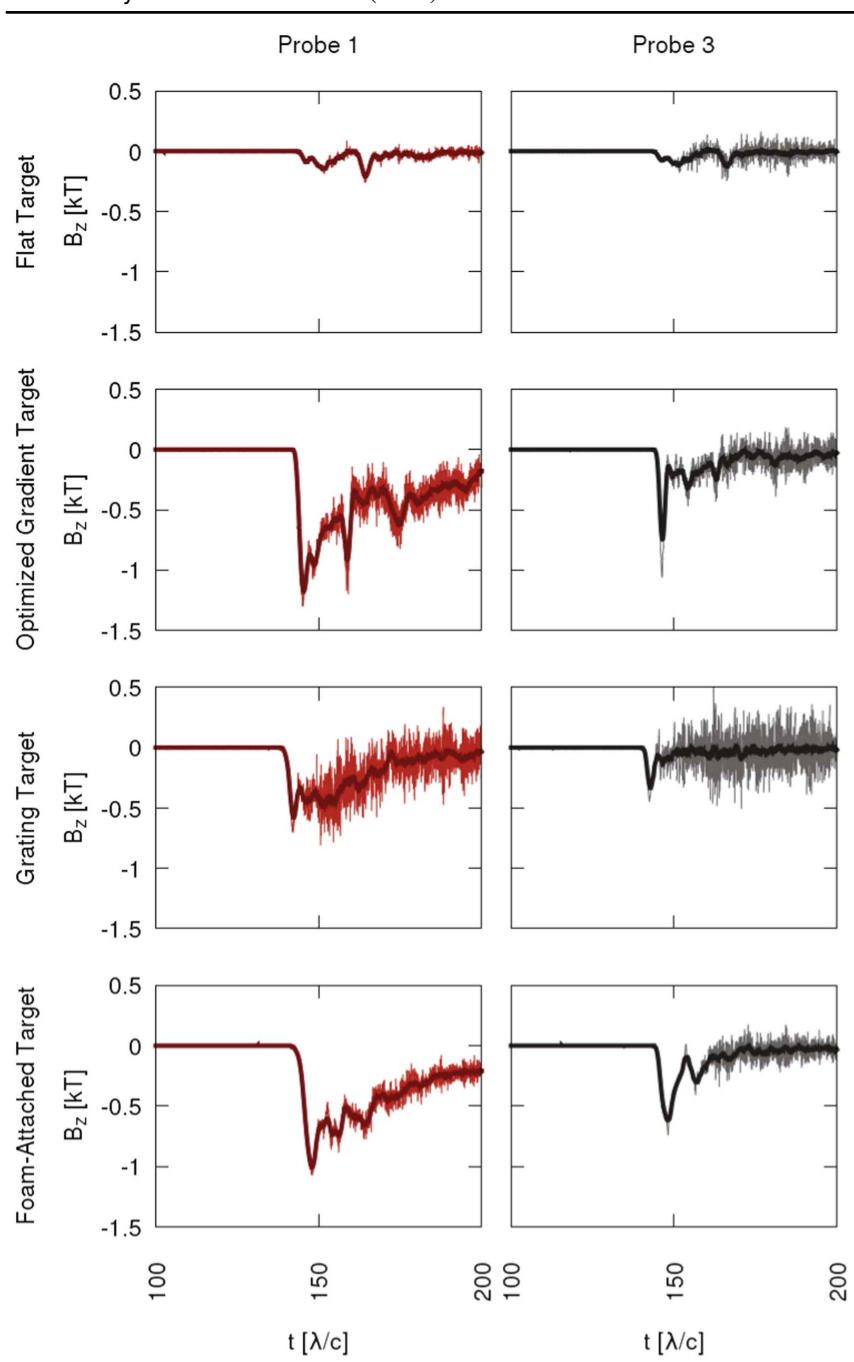

Figure 5. $\hat{z}$ component of the magnetic field as a function of time measured by probes 1 and 3 (see figure 3 ) for all the simulated targets. The light shade curves represent raw data, whereas the darker curves are calculated averaging over 120 neighbouring points (i.e. within $\mathrm{a} \pm 0.86 \lambda / c$ interval). Field intensities are shown in kiloTesla units (for the conversion from code units, a laser wavelength $\lambda=0.8 \mu \mathrm{m}$ was considered).

\section{THz generation with structured targets}

$\mathrm{THz}$ sources are of great interest for several scientific and technological applications [58, 59], such as time-resolved $\mathrm{THz}$ spectroscopy [60] or imaging [61]. Conventional $\mathrm{THz}$ sources with high energy per pulse (in the range of $\sim 100 \mu \mathrm{J}$ ) are based on large electron accelerators [62, 63]. Recently, sources based on ultra-intense laser-solid interaction have been proposed as a possible path to generate $\mathrm{THz}$ pulses with energies similar to those that can be obtained with particle accelerators, but with a compact set-up [6, 12, 64-69]. The experimental observation of $400 \mu \mathrm{J}$ per pulse broadband $\mathrm{THz}$ emission from solid foils irradiated at $I \sim 10^{19} \mathrm{~W} \mathrm{~cm}^{-2}$ has been reported $[6,66]$, with a peak power of the emitted radiation approaching $1 \mathrm{GW}[6]$. THz emission from the rear of irradiated targets is attributed to coherent transition radiation of hot electrons [12, 65, 66, 70, 71], transient surface
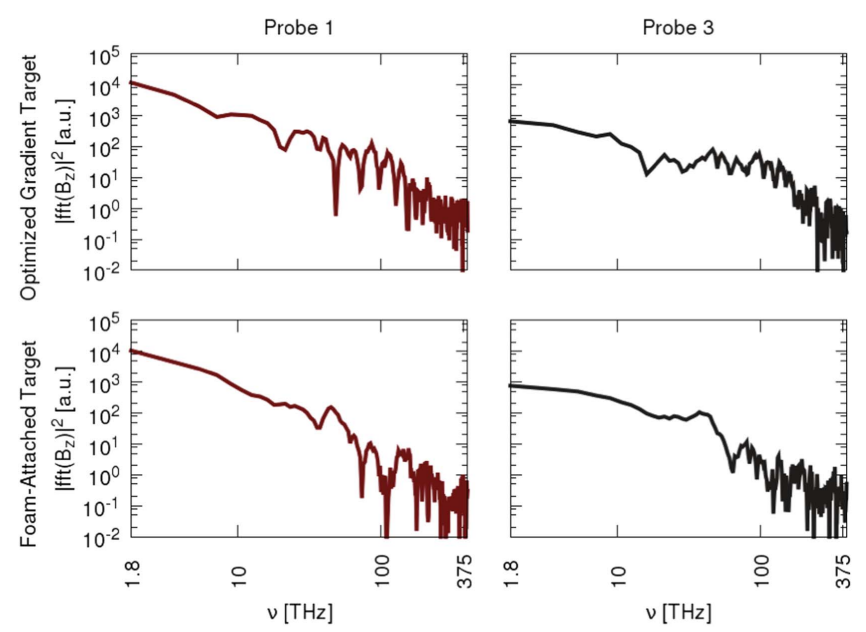

Figure 6. Squared amplitude of the Fourier transform of the $\hat{z}$ component of the magnetic field seen by probes 1 and 3 . The lower limit of the frequency axis $(1.8 \mathrm{THz})$ is due to the time window of the probe diagnostics. The laser frequency is $\approx 375 \mathrm{THz}$. Only data for the optimized gradient target and the foam-attached target are shown, since the strongest emission is observed in these cases.

currents [6] and time varying TNSA charge separation field at the rear side of the target [6]. In these scenarios the intensity of $\mathrm{THz}$ emission has been directly correlated with the laser absorption. As already discussed, structured targets provide a mean to improve laser absorption [11,13-15], which might also lead to an enhancement of $\mathrm{THz}$ emission. Here we present an exploratory numerical investigation of $\mathrm{THz}$ emission from irradiated gratings and multilayer targets. The properties of $\mathrm{THz}$ emission using these structured targets are compared with those obtained using a simple foil and a solid foil with a tailored exponential ramp (the gradient length-scale has been chosen so to enhance resonance absorption, as in [12]).

Numerical simulations were performed with the open source, massively parallel PIC code Smilei [72]. In this section Smilei rather than piccante was used due to its additional diagnostics, such as 'probes' and time-averaged fields. Cross-checks were performed between the two codes showing a very good agreement. A $250 \lambda \times 448 \lambda$ numerical box with a resolution of 48 points per wavelength was used. The simulation time was $350 \lambda / c$. The P-polarized laser pulse had a Gaussian transverse shape (waist of $5 \lambda$ ), a Gaussian temporal profile (field FWHM duration of $15 \lambda / c$ ) and a normalized laser intensity of $a_{0}=2$ (i.e. a mildly relativistic regime accessible with a compact $\sim 10$ TW laser system [14]). The incidence angle was $30^{\circ}$.

Four different targets were simulated: a simple flat foil ( $5 \lambda$ thick, $n_{\mathrm{e}}=40 n_{\mathrm{c}}$ ), a grating target ( $5 \lambda$ thick, $n_{\mathrm{e}}=40 n_{\mathrm{c}}$, $0.25 \lambda$ peak-to-valley depth, distance of the grooves $d=2 \lambda$ ), a foam-attached target (flat foil plus $5 \lambda$ thick uniform foam layer at $n_{\mathrm{e}}=1 n_{\mathrm{c}}$ ) and a simple target with an exponential

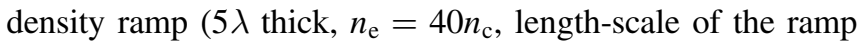
$l=0.65 \lambda$, which is the optimal length-scale for resonance absorption at $\left.30^{\circ}[73]\right)$. Forty-nine macro-electrons per cell were used to sample the electron density.

Figure 3 shows the $\hat{z}$ component (averaged over one laser cycle) of the magnetic field at $t=200 \lambda / c$ for the considered targets. In the four cases the emission of a half-cycle 
electromagnetic burst from the back side of the target can be observed. The lowest intensity of the emitted radiation is observed for the flat target. All the other targets allow for a significant enhancement of electromagnetic emission with respect to the former. The strongest emission is obtained with the gradient target and the foam-attached target. The intensity of this electromagnetic burst can be directly correlated with the conversion efficiency of laser energy into electron kinetic energy, reported in figure 4 . The absorption efficiency is $\approx 10 \%$ for the flat target, while it reaches $\approx 20 \%$ for the grating target and $\approx 60 \%$ for the other two targets. Figure 5 shows the $\hat{z}$ component of the magnetic field measured by 'probes' 1 and 3 (see figure 3 ). The decay time of the signal is of the order of several tens of $\lambda / c$, which implies a typical frequency of few tens of $\mathrm{THz}$ for $\lambda=0.8 \mu \mathrm{m}$.

Figure 6 shows the squared amplitude of the Fourier transform of the signal detected by probes 1 and 3 for the optimized gradient and the foam-attached targets. The signal is broadband between few $\mathrm{THz}$ and $\approx 100 \mathrm{THz}$. Limited differences can be observed in the spectra obtained with the two targets. These results confirmed that, as previously suggested [12], the THz emission from a target with a controlled plasma gradient is significantly higher than from a simple flat foil. The grating target allows for a moderate enhancement of the $\mathrm{THz}$ emission. On the other hand, using foam-attached targets the emission intensity is comparable with that obtained with the gradient target. However, foam-attached targets do not require a fine control of the laser temporal shape nor a pre-heating pulse in order to obtain a tailored gradient. This relaxes the experimental set-up, while still allowing for a significant $\mathrm{THz}$ emission.

\section{Conclusions}

Our results show that structured targets can be used to optimize laser-plasma coupling for a variety of applications. Here we have considered two specific target designs irradiated at laser intensities readily available in existing laser facilities: grating targets and foam-attached targets. Irradiating grating targets with an angle of incidence close to the one expected for surface plasmon resonance allows a significant enhancement of HHG with respect to simple flat targets. Foam-attached targets consisting of solid foils coated with a near-critical layer allow one to strongly enhance the laser-to-electrons conversion efficiency; this leads to an increase of the maximum energy and of the total charge of the TNSA ions. Finally, numerical simulations suggest that gratings and multilayer targets are a promising solution for the generation of electromagnetic bursts at $\mathrm{THz}$ frequencies, since in both cases a strong enhancement of $\mathrm{THz}$ emission with respect to simple flat targets is observed.

\section{Acknowledgments}

We gratefully thank Anna Grassi (Pierre et Marie Curie University, Paris and University of Pisa, Italy) for providing assistance in the use of Smilei PIC code.
The research leading to these results has received funding from the European Research Council Consolidator Grant ENSURE (ERC-2014-CoG No. 647554). We also acknowledge IscraC and LISA access schemes to MARCONI HPC machine at CINECA(Italy) via the projects LAST and SNAP.

\section{References}

[1] Esarey E, Schroeder C B and Leemans W P 2009 Physics of laser-driven plasma-based electron accelerators Rev. Mod. Phys. 81 1229-85

[2] Daido H, Nishiuchi M and Pirozhkov A S 2012 Review of laser-driven ion sources and their applications Rep. Prog. Phys. 75056401

[3] Macchi A, Borghesi M and Passoni M 2013 Ion acceleration by superintense laser-plasma interaction Rev. Mod. Phys. 85 751-93

[4] Sarri G et al 2013 Table-top laser-based source of femtosecond, collimated, ultrarelativistic positron beams Phys. Rev. Lett. 110255002

[5] Teubner U and Gibbon P 2009 High-order harmonics from laser-irradiated plasma surfaces Rev. Mod. Phys. 81 445-79

[6] Gopal A et al 2013 Observation of gigawatt-class THz pulses from a compact laser-driven particle accelerator Phys. Rev. Lett. 111074802

[7] Prencipe I et al 2017 Targets for high repetition rate laser facilities: needs, challenges and perspectives High Power Laser Sci. Eng. 5

[8] Fedeli L, Sgattoni A, Cantono G and Macchi A 2017 Relativistic surface plasmon enhanced harmonic generation from gratings Appl. Phys. Lett. 110051103

[9] Prencipe I et al 2016 Development of foam-based layered targets for laser-driven ion beam production Plasma Phys. Control. Fusion 58034019

[10] Passoni M et al 2016 Toward high-energy laser-driven ion beams: nanostructured double-layer targets Phys. Rev. Accel. Beams 19061301

[11] Cialfi L, Fedeli L and Passoni M 2016 Electron heating in subpicosecond laser interaction with overdense and nearcritical plasmas Phys. Rev. E 94053201

[12] Ding W J and Sheng Z M 2016 Sub GV/cm terahertz radiation from relativistic laser-solid interactions via coherent transition radiation Phys. Rev. E 93063204

[13] Ceccotti T et al 2013 Evidence of resonant surface-wave excitation in the relativistic regime through measurements of proton acceleration from grating targets Phys. Rev. Lett. 111 185001

[14] Blanco M, Flores-Arias M T, Ruiz C and Vranic M 2017 Table-top laser-based proton acceleration in nanostructured targets New J. Phys. 19033004

[15] Sgattoni A, Londrillo P, Macchi A and Passoni M 2012 Laser ion acceleration using a solid target coupled with a lowdensity layer Phys. Rev. E 85036405

[16] Bigongiari A, Raynaud M, Riconda C and Héron A 2013 Improved ion acceleration via laser surface plasma waves excitation Phys. Plasmas 20052701

[17] Pan K Q, Zheng C Y, Wu D, Cao L H, Liu Z J and He X T 2015 Study of strong enhancement of synchrotron radiation via surface plasma waves excitation by particle-in-cell simulations Appl. Phys. Lett. 107183902

[18] Raynaud M, Kupersztych J, Riconda C, Adam J C and Héron A 2007 Strongly enhanced laser absorption and electron acceleration via resonant excitation of surface plasma waves Phys. Plasmas 14092702 
[19] Fedeli L et al 2016 Electron acceleration by relativistic surface plasmons in laser-grating interaction Phys. Rev. Lett. 116 015001

[20] Yeung M, Zepf M, Geissler M and Dromey B 2011 Angularly separated harmonic generation from intense laser interaction with blazed diffraction gratings Opt. Lett. 36 2333-5

[21] Cerchez M, Giesecke A L, Peth C, Toncian M, Albertazzi B, Fuchs J, Willi O and Toncian T 2013 Generation of laserdriven higher harmonics from grating targets Phys. Rev. Lett. 110065003

[22] Lavocat-Dubuis X and Matte J-P 2009 Numerical simulation of harmonic generation by relativistic laser interaction with a grating Phys. Rev. E 80055401

[23] Lavocat-Dubuis X and Matte J-P 2010 Numerical and theoretical study of the generation of extreme ultraviolet radiation by relativistic laser interaction with a grating Phys. Plasmas 17093105

[24] Zhang S J, Zhuo H B, Zou D B, Gan L F, Zhou H Y, Li X Z, Yu M Y and Yu W 2016 Model of high-order harmonic generation from laser interaction with a plasma grating Phys. Rev. E 93053206

[25] Pan K Q, Zheng C Y and He X T 2016 Surface plasma waves with their harmonics generation from pre-structured targets Phys. Plasmas 23023109

[26] von der Linde D, Engers T, Jenke G, Agostini P, Grillon G, Nibbering E, Mysyrowicz A and Antonetti A 1995 Generation of high-order harmonics from solid surfaces by intense femtosecond laser pulses Phys. Rev. A 52 R25-7

[27] Norreys P A et al 1996 Efficient extreme UV harmonics generated from picosecond laser pulse interactions with solid targets Phys. Rev. Lett. 76 1832-5

[28] Bierbach J et al 2012 Generation of $10 \mu \mathrm{W}$ relativistic surface high-harmonic radiation at a repetition rate of $10 \mathrm{~Hz} \mathrm{New} \mathrm{J.}$ Phys. 14065005

[29] an der Brügge D, Kumar N, Pukhov A and Rödel C 2012 Influence of surface waves on plasma high-order harmonic generation Phys. Rev. Lett. 108125002

[30] Kahaly S, Monchocé S, Vincenti H, Dzelzainis T, Dromey B, Zepf M, Martin P and Quéré F 2013 Direct observation of density-gradient effects in harmonic generation from plasma mirrors Phys. Rev. Lett. 110175001

[31] Baeva T, Gordienko S and Pukhov A 2006 Theory of highorder harmonic generation in relativistic laser interaction with overdense plasma Phys. Rev. E 74046404

[32] Quéré F, Thaury C, Monot P, Dobosz S, Martin P, Geindre J-P and Audebert P 2006 Coherent wake emission of high-order harmonics from overdense plasmas Phys. Rev. Lett. 96125004

[33] Paul A, Bartels R A, Tobey R, Green H, Weiman S, Christov I P, Murnane M M, Kapteyn H C and Backus S 2003 Quasi-phase-matched generation of coherent extremeultraviolet light Nature 421 51-4

[34] Yeung M et al 2013 Near-monochromatic high-harmonic radiation from relativistic laser plasma interactions with blazed grating surfaces New J. Phys. 15025042

[35] Sgattoni A, Fedeli L, Cantono G, Ceccotti T and Macchi A 2016 High field plasmonics and laser-plasma acceleration in solid targets Plasma Phys. Control. Fusion 58014004

[36] Pitarke J M, Silkin V M, Chulkov E V and Echenique P M 2007 Theory of surface plasmons and surface-plasmon polaritons Rep. Prog. Phys. 701

[37] Sgattoni A, Fedeli L and Sinigardi S Piccante: an open-source, fully relativistic, massively parallel particle-in-cell code http://aladyn.github.io/piccante/

[38] Sgattoni A, Fedeli L, Sinigardi S, Marocchino A, Macchi A, Weinberg V and Karmakar A 2015 Optimising PICCANTE -an open source particle-in-cell code for advanced simulations on Tier-0 systems (arXiv:1503.02464)
[39] Okihara S et al 2004 Ion generation in a low-density plastic foam by interaction with intense femtosecond laser pulses Phys. Rev. E 69026401

[40] Zani A, Dellasega D, Russo V and Passoni M 2013 Ultra-low density carbon foams produced by pulsed laser deposition Carbon 56 358-65

[41] Bin J H et al 2015 Ion acceleration using relativistic pulse shaping in near-critical-density plasmas Phys. Rev. Lett. 115064801

[42] Willingale L et al 2009 Characterization of high-intensity laser propagation in the relativistic transparent regime through measurements of energetic proton beams Phys. Rev. Lett. 102125002

[43] Passoni M, Zani A, Sgattoni A, Dellasega D, Macchi A, Prencipe I, Floquet V, Martin P, Liseykina T V and Ceccotti T 2014 Energetic ions at moderate laser intensities using foam-based multi-layered targets Plasma Phys. Control. Fusion 56045001

[44] Najmudin Z, Fiuza F and Fernndez J C 2016 Summary of working group 6: ion acceleration with lasers AIP Conf. Proc. 1777030006

[45] Linz U and Alonso J 2016 Laser-driven ion accelerators for tumor therapy revisited Phys. Rev. Accel. Beams 19124802

[46] Bulanov S V, Zh Esirkepov T, Khoroshkov V S, Kuznetsov A V and Pegoraro F 2002 Oncological hadrontherapy with laser ion accelerators Phys. Lett. A 299 240-7

[47] Amato E, Italiano A, Margarone D, Pagano B, Baldari S and Korn G 2016 Future laser-accelerated proton beams at elibeamlines as potential source of positron emitters for PET J. Instrum. $11 \mathrm{C} 04007$

[48] Hidding B et al 2017 Laser-plasma-based space radiation reproduction in the laboratory Sci. Rep. 742354

[49] Barberio M, Veltri S, Scisciò M and Antici P 2017 Laseraccelerated proton beams as diagnostics for cultural heritage Sci. Rep. 740415

[50] Alejo A, Ahmed H, Green A, Mirfayzi S R, Borghesi M and Kar S 2016 Recent advances in laser-driven neutron sources Nuovo Cimento C 38188

[51] Nakamura T, Tampo M, Kodama R, Bulanov S V and Kando M 2010 Interaction of high contrast laser pulse with foam-attached target Phys. Plasmas 17113107

[52] Pukhov A and Meyer-ter Vehn J 1996 Relativistic magnetic selfchanneling of light in near-critical plasma: three-dimensional particle-in-cell simulation Phys. Rev. Lett. 76 3975-8

[53] Gahn C, Tsakiris G D, Pukhov A, Meyer-ter Vehn J, Pretzler G, Thirolf P, Habs D and Witte K J 1999 Multi$\mathrm{MeV}$ electron beam generation by direct laser acceleration in high-density plasma channels Phys. Rev. Lett. 83 4772-5

[54] Thaury C et al 2007 Plasma mirrors for ultra-high intensity optics Nat. Phys. 3 424-9

[55] Danson C, Hillier D, Hopps N and Neely D 2015 Petawatt class lasers worldwide High Power Laser Sci. Eng. 3 e3

[56] Fedeli L, Formenti A, Bottani C E and Passoni M 2017 Parametric investigation of laser interaction with uniform and nanostructured near-critical plasmas Eur. Phys. J. D 18202

[57] Passoni M and Lontano M 2008 Theory of light-ion acceleration driven by a strong charge separation Phys. Rev. Lett. 101115001

[58] Ferguson B and Zhang X-C 2002 Materials for terahertz science and technology Nat. Mater. 1 26-33

[59] Hafez H A, Chai X, Ibrahim A, Mondal S, Frachou D, Ropagnol X and Ozaki T 2016 Intense terahertz radiation and their applications J. Opt. 18093004

[60] Ulbricht R, Hendry E, Shan J, Heinz T F and Bonn M 2011 Carrier dynamics in semiconductors studied with timeresolved terahertz spectroscopy Rev. Mod. Phys. 83 543-86

[61] Chan W L, Deibel J and Mittleman D M 2007 Imaging with terahertz radiation Rep. Prog. Phys. 701325 
[62] Stojanovic N and Drescher M 2013 Accelerator- and laserbased sources of high-field terahertz pulses J. Phys. B: At. Mol. Opt. Phys. 46192001

[63] Wu Z, Fisher A S, Goodfellow J, Fuchs M, Daranciang D, Hogan M, Loos H and Lindenberg A 2013 Intense terahertz pulses from SLAC electron beams using coherent transition radiation Rev. Sci. Instrum. 84022701

[64] Ding W J, Sheng Z M and Koh W S 2013 High-field half-cycle terahertz radiation from relativistic laser interaction with thin solid targets Appl. Phys. Lett. 103204107

[65] Liao G Q et al 2015 Bursts of terahertz radiation from largescale plasmas irradiated by relativistic picosecond laser pulses Phys. Rev. Lett. 114255001

[66] Liao G Q et al 2017 Intense terahertz radiation from relativistic laser plasma interactions Plasma Phys. Control. Fusion 59 014039

[67] Tian Y, Liu J, Bai Y, Zhou S, Sun H, Liu W, Zhao J, Li R and Xu Z 2017 Femtosecond-laser-driven wire-guided helical undulator for intense terahertz radiation Nat. Photon. 11 242-6

[68] Nakajima K 2017 Novel efficient THz undulator using a laserdriven wire Light Sci. Appl. 6 e17063

[69] Mondal S et al 2017 Aligned copper nanorod arrays for highly efficient generation of intense ultra-broadband thz pulses Sci. Rep. 740058

[70] Jin Z et al 2016 Highly efficient terahertz radiation from a thin foil irradiated by a high-contrast laser pulse Phys. Rev. E 94 033206

[71] Liao G-Q et al 2016 Demonstration of coherent terahertz transition radiation from relativistic laser-solid interactions Phys. Rev. Lett. 116205003

[72] Derouillat J et al 2017 SMILEI: a collaborative, open-source, multi-purpose particle-in-cell code for plasma simulation (arXiv:1702.05128)

[73] Gibbon P 2004 Short Pulse Laser Interactions with Matter (Singapore: World Scientific) 